\title{
FIELDS WITH FEW TYPES
}

\author{
CÉDRIC MILLIET
}

\begin{abstract}
According to Belegradek, a first order structure is weakly small if there are countably many 1-types over any of its finite subset. We show the following results. A field extension of finite degree of an infinite weakly small field has no Artin-Schreier extension. A weakly small field of characteristic 2 is finite or algebraically closed. A weakly small division ring of positive characteristic is locally finite dimensional over its centre. A weakly small division ring of characteristic 2 is a field.
\end{abstract}

In model theory, much attention has been drawn on algebraic structures which lie on the classifiable part of the dividing line provided by Shelah's stability theory, following the criterion: the models of some fixed uncountable cardinality of a theory $T$ should not be classifiable if there are too many of them, such as if $T$ is unstable, or stable non-superstable. Concerning theories with few countable models, less is known, even for $\aleph_{0}$-categorical groups (who have only one countable model up to isomorphism). All the less for the so called small structures who include all possible theories having fewer than continuum many countable models. Known results about small structures concern type-definable equivalence relations $[15,8,9]$, abelian groups [21], fields [24] and classes of modules [20]. Some have been studied under additional model-theoretic hypothesis: small stable groups [21] and more generally $\mathfrak{R}$-groups [23, chapter 5$]$, $\aleph_{0}$-categorical supersimple groups [3], small one-based structures [14] and small structures with finite coding [5].

Weakly small structures are introduced by Belegradek in [24] to provide a broad framework for both small and minimal structures: they include omega-stable structures but also $\aleph_{0}$-categorical, minimal, and $d$-minimal ones recently introduced by Poizat in [19].

In [24], Wagner shows that infinite small fields are algebraically closed, making the first successful use of the Cantor-Bendixson rank (the very weak analogue of Morley rank) in an algebraic context. He asks whether infinite weakly small fields are algebraically closed [24, Problem 12.5]. Following ideas of [24], an exploration of weakly small groups begins in [13] where it is noticed that a weakly small group $G$ inherits locally several properties that omega-stable groups share globally. For instance $G$ satisfies local descending chain conditions. Every definable subset of

Received April 1, 2011.

2010 Mathematics Subject Classification. 03C45, 03C60.

Key words and phrases. Small, weakly small, field, Artin-Schreier extension, Cantor-Bendixson rank, local descending chain condition.

Most of this paper forms part of the author's doctoral dissertation, written in Lyon under the supervision of Wagner. Many thanks to Poizat and Point for rewarding comments and references. 
$G$ has a local stabiliser with good local properties. If $G$ is infinite, it possesses an infinite abelian subgroup, not necessarily definable though.

Let us bring to mind what is known about weakly small fields. An $\aleph_{0}$-categorical field is finite. Macintyre showed in 1971 that an omega-stable field is either finite or algebraically closed [11]. Wagner drew the same conclusion for a small field, as well as for a minimal field of positive characteristic $[24,25]$. Poizat extended the latter to $d$-minimal fields of positive characteristic [19]. Whether the same result holds even for a minimal field of characteristic zero is still unknown.

We begin Section 2 by giving another proof that an infinite small field is algebraically closed. In section 3 , we make a first significant step towards [24, Problem 12.5]:

THEOREM 0.1. If $K$ is an infinite weakly small field, and $L / K$ a field extension of finite degree, then $L$ has no Artin-Schreier extensions.

Using the classification of finite simple groups, it follows that:

COROLLARY 0.2. A weakly small field of characteristic 2 is finite or algebraically closed.

Wagner had already noticed in [24] that a weakly small field is either finite or has no Artin-Schreier nor Kummer extensions. As a field extension of finite degree of a weakly small field has no obvious reason to be weakly small, Theorem 0.1 is a non-trivial improvement of [24].

In section 4, we do not assume commutativity anymore and show that a weakly small division ring of positive characteristic is locally finite dimensional over its centre. We derive:

COROLlary 0.3. A weakly small division ring of characteristic 2 is a field.

Recall that superstable division rings [1, Cherlin, Shelah] and even supersimple ones [16, Pillay, Scanlon, Wagner] are fields; so are small division rings of positive characteristic [12].

\section{$\S 1$. Preliminaries: weakly small tools.}

Definition 1.1. A theory is small if it has countably many complete $n$-types without parameters (or equivalently over any fixed finite parameter set) for every natural number $n$. A structure is small if so is its theory.

DEFINITION 1.2 (Belegradek). A structure is weakly small if for any of its finite subsets $A$, there are countably many complete l-types over $A$.

For convenience of the reader, we state here the main results of [13] that will be needed in the sequel. We refer to the latter paper the reader willing to know more about weakly small groups.

In a weakly small structure $M$, for any finite parameter subset $A$ of $M$, the space $S_{1}(A)$ of complete 1-types over $A$ is a countable compact Hausdorff space. It has an ordinal Cantor-Bendixson rank and one can compute the Cantor-Bendixson rank over $A$ of any of its element $p$. We write it $C B_{A}(p)$. For any $A$-definable set $X$ of arity 1, we write $C B_{A}(X)$ for the maximum Cantor rank of the complete 1-types over $A$ containing the formula defining $X$. Only a finite number of complete 1-types over $A$ with same $C B_{A}$-rank as $X$ do contain the formula defining $X$. We call this natural number the Cantor-Bendixson degree of $X$ over $A$, and write it $d C B_{A}(X)$. 
What has been said for 1-types of a weakly small structure is also valid for every $n$-type of a small structure over parameters in an arbitrary finite set. The following two Lemmas hold in any structure.

LEMMA 1.3. Let $X$ and $Y$ be A-definable sets. Let $f$ be an A-definable map from $X$ onto $Y$. If the fibres of $f$ have no more than $n$ elements, then $f$ preserves the Cantor rank over $A$. Moreover,

$$
d C B_{A}(X) \leq d C B_{A}(Y) \leq n \cdot d C B_{A}(X) .
$$

LEMMA 1.4. Let $X$ be a $\emptyset$-definable set, and a an element algebraic over the empty set. Then $C B_{a}(X)$ equals $C B_{\emptyset}(X)$.

Lemma 1.4 allows to define the local Cantor rank of an $a$-definable set $X$, to be its Cantor rank over any $b$ defining $X$ and having the same algebraic closure as $a$. We shall write $\operatorname{acl}(B)$ for the algebraic closure of some set $B$, and $\operatorname{dcl}(B)$ for its definable closure.

THEOREM 1.5 (Weakly small descending chain condition). In a weakly small group, the trace over acl $(\emptyset)$ of a descending chain of acl $(\emptyset)$-definable subgroups becomes stationary after finitely many steps.

For any $\emptyset$-definable set $X$ in a weakly small group $G$, if $\Gamma$ is the algebraic closure of a finite tuple $g$ from $G$, one can define the local almost stabiliser of $X$ in $\Gamma$ to be

$$
\operatorname{Stab}_{\Gamma}(X)=\left\{x \in \Gamma: C B_{x, g}(x X \Delta X)<C B_{g}(X)\right\} .
$$

$S t a b_{\Gamma}(X)$ is a subgroup of $\Gamma$. If $\delta$ is any subgroup of $\Gamma$, we write $\operatorname{Stab}_{\delta}(X)$ for $\operatorname{Stab}_{\Gamma}(X) \cap \delta$. Here is a local analogue of what happens for the stabiliser of a definable set of maximal Morley rank in an omega-stable structure:

PROPOSITION 1.6. Let $G$ be a weakly small group, $g$ a finite tuple of $G$, and $X a$ $g$-definable subset of $X$. If $\delta$ is a subgroup of $d c l(g)$ and if $X$ has maximal Cantor rank over $g$, then $\operatorname{Stab}_{\delta}(X)$ has finite index in $\delta$.

Next proposition can be found in [12].

Proposition 1.7. Let $G$ be a small group, and $f$ a definable group homomorphism of $G$. There exists a natural number $n$ such that $\operatorname{Ker} f^{n} \cdot \operatorname{Im} f^{n}$ equals $G$.

§2. Weakly small fields. We begin by proposing a simplified proof of a result from Wagner [24].

LEMMA 2.1. An infinite weakly small field is perfect.

Proof. Let $f$ be a group homomorphism of some weakly small group G. Suppose that $f$ has finite kernel of cardinal $n$. An easy consequence of Lemma 1.3 is that the image of $f$ has index at most $n$ in $G$. It follows that the image of the Frobenius map is a sub-field having finite additive (and multiplicative!) index. $\dashv$

THEOREM 2.2. A weakly small infinite field, possibly skew, has no definable additive nor multiplicative subgroup of finite index.

Proof. Let $K$ be this field and let $H$ be a definable additive subgroup of $K$ having finite index. Suppose first that there is an infinite finitely generated algebraic closure $\Gamma$. Note that $\Gamma$ is a field. The intersection of $\lambda H \cap \Gamma$ where $\lambda$ runs over $\Gamma$ is a finite intersection by Theorem 1.5 hence has finite index in $\Gamma$. It is also an ideal of $\Gamma$ and 
must equal $\Gamma$. So $\Gamma$ is included in $H$. Since this also holds for any finitely generated algebraic closure containing $\Gamma$, the group $H$ equals $K$.

Otherwise, $K$ is locally finite. By Wedderburn's theorem, $K$ is commutative and equals $\operatorname{acl}(\emptyset)$. According to Theorem 1.5, it satisfies the descending chain condition on definable subgroups. $K$ has a smallest definable additive subgroup of finite index, which must be an ideal, and hence equals $K$.

For the multiplicative case now, let us consider a multiplicative subgroup $M$ of $K^{\times}$having index $n$. Let us suppose first that there is $\delta$ an infinite finitely generated sub-field of $K$. There is no harm in extending $\delta$ so that each coset of $M$ be $\delta$ definable. $M$ has maximal Cantor rank over $\delta$ by Lemma 1.3, so its almost additive stabiliser in $\delta$ has finite additive index in $\delta$ by Proposition 1.6, as well as the almost stabiliser of any of its cosets. So the almost stabiliser of all the cosets is an ideal of $\delta$ having finite index, hence equals the whole of $\delta$. We finish as Poizat in [19] by showing that the complement of $M$ has small Cantor rank: we have just shown that $1+a M \simeq a M$ for every coset $a M$, where $\simeq$ stands for equality up to small Cantor rank over $\delta$. For every coset $a M$, and every $x$ in $a M$ but a small ranked set, $1+x$ belongs to $a M$, so $x^{-1}+1 \in M$ and $x^{-1} \in M-1$.

Otherwise $K$ is locally finite and has characteristic $p$. By Lemma 2.1, the group $K^{\times}$is $p$-divisible, so $K^{\times}$can not have a proper subgroup of finite index.

Before going further, we remind a few definitions. Let $L / K$ be a field extension. It is a Kummer extension if it is generated by $K$ and one $n$th root of some element in $K$. It is an Artin-Schreier extension, if $L$ is generated by $K$ and one $x$ such that $x^{p}-x$ belongs to $K$ ( $x$ is called a pseudo-root of $K$ ).

FACT 2.3 (Artin-Schreier, Kummer [10]). Let $K$ be a field of characteristic $p$ (possibly zero) and $L$ a cyclic Galois extension of finite degree $n$.

(i) Suppose that $p$ is zero, or coprime with $n$. If $K$ contains $n$ distinct $n$th roots of 1 , then $L / K$ is a Kummer extension.

(ii) If $p$ equals $n$, then $L / K$ is an Artin-Schreier extension.

COROLLARY 2.4 (Wagner [24]). A small field is finite or algebraically closed.

Proof. Let $K$ be a small infinite field. For every natural number $n$, the $n$th power map has bounded fibres so its image has finite index by Lemma 1.3, and the map is onto by Theorem 2.2: $K^{\times}$is divisible. By the same argument, the map mapping $x$ to $x^{p}-x$ is onto. We conclude as Macintyre in [11] for omega-stable fields: first of all, $K$ contains every root of unity. For if $a$ is a $n$th root of 1 not in $K$ with minimal order, $K(a) / K$ has degree $m<n$. By minimality of $n$, every $m$ th root of unity is in $K$. If $m$ is zero or coprime with the characteristic of $K$, then $K(a)$ is of the form $K(b)$ with $b^{m} \in K$ after Fact 2.3. As $K^{\times}$is divisible, $b$ is in $K$, a contradiction. So $K$ has positive characteristic $p$, and $p$ divides $m . K(a)$ contains an extension of degree $p$ over $K$, which is an Artin-Schreier extension after Fact 2.3, a contradiction.

Secondly, if $K$ is not algebraically closed, it has a normal extension $L$ of finite degree $n$, which must be separable as $K$ is perfect (Lemma 2.1); its Galois group contains a cyclic sub-group of prime order $q$, the invariant field of which we call $M$. Note that $L$ is interpretable in a finite Cartesian power of $K$, so is small too. If $q$ is not the characteristic, as $K$ contains every $q$ th root of 1 , the extension $L / M$ is Kummer; if $q$ equals the characteristic, $L / M$ is an Artin-Schreier extension, a contradiction in both cases. 
$\S 3$. Weakly small fields. The first part of the previous proof still holds for weakly small fields:

COROLLARY 3.1. An infinite weakly small field has no Galois solvable extension.

Proof. If $L / K$ is a Galois solvable extension, there is a tower of fields $K=$ $K_{0} \subset K_{1} \subset \cdots \subset K_{n}=L$ so that each $K_{i+1} / K_{i}$ be generated by either a $n$th root or a pseudo-root of some element $K_{i}$. But $K_{0}$ has no Artin-Schreier or Kummer extension by the proof of Corollary 2.4 .

Note however that, as an algebraic extension of a weakly small field has no obvious reason to be weakly small, we cannot apply Macintyre's argument to deduce that a weakly small field is finite or algebraically closed. Nevertheless, stepping on the additive structure of the field, we can show that every algebraic extension of finite degree of an infinite weakly small field is Artin-Schreier closed. This is a first step towards Problem 12.5 in [24] asking whether an infinite weakly small field is algebraically closed.

Corollary 3.16 was conjectured by Poizat, who suggested using weakly normal groups and [6, Theorem 4.1]. We ended up using the closely related Abelian structures and Fact 3.3 below.

DefinItION 3.2. An Abelian structure is any abelian group together with predicates interpreting subgroups of its finite Cartesian powers.

As for a pure module, an Abelian structure has quantifier elimination up to positive prime formulas (see [26, Weispfenning], or [23, Theorem 4.2.8]):

FACT 3.3 (Weispfenning). In an Abelian structure $A$, a definable set is a boolean combination of cosets of acl $(\emptyset)$-definable subgroups of Cartesian powers of $A$.

An Abelian structure $A$ is stable: let us take a formula $\varphi(x, y)$ such that $\varphi(x, 0)$ defines an $\operatorname{acl}(\emptyset)$-definable subgroup of $A^{n}$, and $\varphi(x, y)$ the coset of $y$. Two formulas $\varphi(x, a)$ and $\varphi(x, b)$ define cosets of the same group, so they must be equal or disjoint. It follows that there are countably many $\varphi$-types over any countable set of parameters. By Fact 3.3, this is sufficient to show that $A$ is stable.

In a stable structure, we call a dense forking chain any chain of complete types $p_{q}$ indexed by $Q$ such that for every rational numbers $q<r$, the type $p_{r}$ be a forking extension of $p_{q}$.

Stable theories with no dense forking chains have been introduced in [14, Pillay]. They generalise superstable ones. In a stable structure $M$ with no dense forking chains, every complete type (and not only 1-types !) has an ordinal dimension, and for any dimension $\alpha$, a Lascar $\alpha$-rank. We shall write $\operatorname{dim}(p)$ for the dimension of the type $p$, and $U_{\alpha}(p)$ for its $\alpha$-rank. They are defined as follows:

Definition 3.4 (Pillay). For two complete types $p \subset q$, let us define the dimension of $p$ over $q$ written $\operatorname{dim}(p / q)$ by the following induction.

1. $\operatorname{dim}(p / q)$ is -1 if $q$ is a nonforking extension of $p$.

2. $\operatorname{dim}(p / q)$ is at least $\alpha+1$ if there are non forking extensions $p^{\prime}$ and $q^{\prime}$ of $p$ and $q$, and infinitely many complete types $p_{1}, p_{2}, \ldots$ such that $p^{\prime} \subset p_{1} \subset p_{2} \subset$ $\cdots \subset q^{\prime}$ and $\operatorname{dim}\left(p_{i} / p_{i+1}\right) \geq \alpha$ for all natural number $i$.

3. $\operatorname{dim}(p / q)$ is at least $\lambda$ for a limit ordinal $\lambda$ if $\operatorname{dim}(p / q) \geq \alpha$ for all $\alpha<\lambda$. 
Definition 3.5 (Pillay). For a complete type $p$, we set $\operatorname{dim}(p)=\operatorname{dim}(p / q)$ where $q$ is any algebraic extension of $p$.

Definition 3.6 (Pillay). For every ordinal $\alpha$, we define inductively the $U_{\alpha}$-rank of a complete type $p$ by

1. $U_{\alpha}(p)$ is at least 0 .

2. $U_{\alpha}(p)$ is at least $\beta+1$ if there is an extension $q$ of $p$ such that $\operatorname{dim}(p / q) \geq \alpha$ and $U_{\alpha}(q) \geq \beta$.

3. $U_{\alpha}(p)$ is at least $\lambda$ for a limit ordinal $\lambda$ if $U_{\alpha}(p) \geq \beta$ for all $\beta<\lambda$.

To any type-definable stable group in $M$ can be associated the $U_{\alpha}$-rank and the dimension of any of its generic types over $M$. We refer the reader to [14, 5, Herwig, Loveys, Pillay, Tanović, Wagner] for more details. We shall just recall two facts: the Lascar inequalities which are still valid for the $U_{\alpha}$-rank, as well as their group version ; and the link between the $U_{\alpha}$-rank and the existence of a dense forking chain.

FACT 3.7 (Lascar inequalities for $U_{\alpha}$-rank $[14,5]$ ).

1. In a stable structure, for every tuple $a, b$ and every set $A$,

$$
U_{\alpha}(b / A a)+U_{\alpha}(a / A) \leq U_{\alpha}(a b / A) \leq U_{\alpha}(b / A a) \oplus U_{\alpha}(a / A) .
$$

2. For any type-definable group $G$ in a stable structure, and any type-definable subgroup $H$ of $G$,

$$
U_{\alpha}(H)+U_{\alpha}(G / H) \leq U_{\alpha}(G) \leq U_{\alpha}(H) \oplus U_{\alpha}(G / H) .
$$

Proof. We only prove point (2), which does not appear anywhere to the author's knowledge but follows from (1). Note that passing from the ambient structure $M$ to $M^{\text {heq }}$, one can use hyperimaginary parameters in (1).

Let $t p(a / M)$ be a generic type of $G$. We write $a_{H}$ the hyperimaginary element which is the image of $a$ in $G / H$. The type $t p\left(a_{H} / M\right)$ is also a generic of $G / H$. Let $b$ be in the connected component $H^{0}$ of $H$, and generic over $M \cup\{a\}$. So $a b$ is a generic of $a H$ over $M \cup\{a\}$, hence over $M \cup\left\{a_{H}\right\}=M \cup\left\{(a b)_{H}\right\}$. As $a$ and $a b$ are in the same class modulo $G^{0}$, they realise the same generic type over $M$. It follows that $t p\left(a / M, a_{H}\right)$ is a generic of $a H$. Now apply point (1) taking some generic of $G$ for $a$ and $b=a_{H}$.

See [5, Lemma 7] and [5, Remark 9] for

FACT 3.8. Let $p$ be a complete n-type. There is a dense forking chain of n-types containing $p$ if and only if the rank $U_{\alpha}(p)$ is not ordinal for every ordinal $\alpha$.

In a $\kappa$-saturated stable structure $M$, for any formula $\varphi(x, y)$, we can compute the Cantor-Bendixson rank of the topological space $S_{\varphi}(M)$ whose elements are the complete $\varphi$-types over $M$. Let $\psi(x)$ be another formula and $S_{\varphi, \psi}$ the subset of $S_{\varphi}(M)$ whose elements are consistent with $\psi$. It is a closed subset of $S_{\varphi}(M)$. The local $\varphi$-rank of $\psi$ is the Cantor-Bendixson rank of $S_{\varphi, \psi}$. We write it $C B_{\varphi}(\psi)$. The local $\varphi$-rank of a type $p$ is the minimum local $\varphi$-rank of the formulas implied by $p$. If $M$ is a stable group, the stratified $\varphi$-rank of $\psi$ is its $\phi$-rank, where $\phi(x, \bar{y})$ stands for the formula $\varphi\left(y_{2} \cdot x, y_{1}\right)$. We write it $C B_{\varphi}^{*}(\psi)$.

In a $\kappa$-saturated stable group $G$, let $H$ and $L$ be two type-definable subgroups. $H$ and $L$ are commensurable if the index of their intersection is bounded (i.e., less 
than $\kappa$ ) in both of them. Recall that this is equivalent to $H$ and $L$ having the same stratified $\varphi$-rank for every formula $\varphi$.

THEOREM 3.9. Let be an Abelian structure with weakly small universe. Its theory has no dense forking chain.

Remark 3.10. Pillay showed that a small 1-based structure has no dense forking chain [14, Lemma 2.1]. In particular, a small Abelian structure has no dense forking chain either. The difficulty of Theorem 3.9 comes from the fact that weak smallness does not bound a priori the number of pure $n$-types for $n \geq 2$, which is a crucial assumption in the proof of [14, Lemma 2.1].

Proof. According to Fact 3.8, one just needs to show that for every finite tuple $\bar{a}$ and set $A$, there is an ordinal $\alpha$ such that $U_{\alpha}(\bar{a} / A)$ is ordinal. Note that the first of Lascar inequalities for the $U_{\alpha}$-rank implies that $U_{\alpha}\left(\left(a_{1}, \ldots, a_{n}\right) / A\right)$ is less than or equal to $U_{\alpha}\left(\left(a_{2}, \ldots, a_{n}\right) / A a_{1}\right) \oplus U_{\alpha}\left(a_{1} / A\right)$. So, by induction on the arity of $\bar{a}$, and Fact 3.8 again, we may consider only 1-types, and suppose for a contradiction that there be a dense forking chain of arity 1.

(1) We first claim that there exists a dense ordered chain $\left(H_{i}\right)_{i \in Q}$ of acl $(\emptyset)$-typedefinable pairwise non commensurable subgroups.

Let $\left(t p\left(a / A_{i}\right)\right)_{i \in Q}$ be a dense forking chain, that is $A_{i}$ is included in $A_{j}$ and $t p\left(a / A_{j}\right)$ forks over $A_{i}$ for all $i<j$. By Fact 3.3, every formula appearing in $\operatorname{tp}\left(a / A_{i}\right)$ is a boolean combination of cosets of $\mathrm{acl}(\emptyset)$-definable groups. There is a smallest $\operatorname{acl}(\emptyset)$-type-definable group $H_{i}$ such that the type $\operatorname{tp}\left(a / A_{i}\right)$ contains the formulas defining $a H_{i}$. If $C B_{\varphi}^{*}\left(a / A_{i}\right)<C B_{\varphi}^{*}\left(a H_{i}\right)$ for some formula $\varphi$, then there is an $\operatorname{acl}(\emptyset)$-definable subgroup $G_{i}$ with the formula defining $a G_{i}$ included in $t p\left(a / A_{i}\right)$, and $C B_{\varphi}^{*}\left(a G_{i}\right)<C B_{\varphi}^{*}\left(a H_{i}\right)$. This implies $C B_{\varphi}^{*}\left(G_{i}\right)<C B_{\varphi}^{*}\left(H_{i}\right)$ and contradicts the minimality of $H_{i}$. It follows that $t p\left(a / A_{i}\right)$ is a generic type of $a H_{i}$. Moreover, $a H_{i}$ is $A_{i}$-type-definable. For all $i<j$, the type $t p\left(a / A_{j}\right)$ forks over $A_{i}$ so there must be a formula $\varphi$ such that $a H_{j}$ and $a H_{i}$ have different stratified $\varphi$ ranks. Then, one has $C B_{\varphi}^{*}\left(H_{i}\right)<C B_{\varphi}^{*}\left(H_{j}\right)$ so $H_{i}$ and $H_{j}$ are non-commensurable groups.

(2) Let us now build $2^{\aleph_{0}}$ complete 1-types over $\emptyset$.

As the structure is stable, each $H_{i}$ is the intersection of decreasing $\operatorname{acl}(\emptyset)$-definable groups $H_{i j}$. Let $\overline{H_{i j}}$ stand for the $\emptyset$-definable finite union of the conjugates of $H_{i j}$ under $A u t(\emptyset)$. Let us call $\widehat{H_{i}}$ the $\emptyset$-type-definable intersection of the (decreasing) $\overline{H_{i j}}$ over $j$. Note that $\widehat{H_{i}}$ is a union of type-definable groups, whose generic types we call the generic types of $\widehat{H_{i}}$. For every real number $r$, we call $p_{r}^{\prime}$ the partial type defining $\bigcap_{i \geq r} \widehat{H}_{i}$ and $p_{r}$ the following partial type

$$
p_{r}^{\prime} \cup\left\{\psi: \psi \text { formula over } \emptyset \text { with } C B_{\varphi}^{*}(\neg \psi)<C B_{\varphi}^{*}\left(p_{r}^{\prime}\right) \text { for some } \varphi\right\}
$$

Note that every formula $\psi$ in the second part of the type $p_{r}$ above is contained in every generic type of the structure (and also in the generic types of $\bigcap_{i \geq r} \widehat{H_{i}}$ ). Every $p_{r}$ is thus consistent. We claim that if $r \neq q$, then $p_{r}$ and $p_{q}$ are inconsistent. In any stable group, if $D_{1}, D_{2}, \ldots$ are decreasing definable subsets, for every formula $\varphi$, there is an index $i_{\varphi}$ such that the equality $C B_{\varphi}^{*}\left(\bigcap_{i \geq 1} D_{i}\right)=C B_{\varphi}^{*}\left(D_{j}\right)$ holds for all $j>i_{\varphi}$. So one can find two indexes $i$ and $j$ (depending on $\varphi$ ) such that all the 
following equalities hold

$$
\begin{aligned}
& C B_{\varphi}^{*}\left(\bigcap_{i \geq r} \widehat{H_{i}}\right)=C B_{\varphi}^{*}\left(\widehat{H_{i}}\right)=C B_{\varphi}^{*}\left(\overline{H_{i j}}\right), \\
& C B_{\varphi}^{*}\left(\bigcap_{i \geq r} H_{i}\right)=C B_{\varphi}^{*}\left(H_{i}\right)=C B_{\varphi}^{*}\left(H_{i j}\right) .
\end{aligned}
$$

As the stratified $\varphi$-ranks are preserved under automorphisms, and as the rank of a finite union equals the maximum of the ranks, $C B_{\varphi}^{*}\left(H_{i j}\right)$ equals $C B_{\varphi}^{*}\left(\overline{H_{i j}}\right)$ and we get

$$
C B_{\varphi}^{*}\left(\bigcap_{i \geq r} \widehat{H_{i}}\right)=C B_{\varphi}^{*}\left(\bigcap_{i \geq r} H_{i}\right)
$$

Recall from point (1) that the groups $H_{i}$ are pairwise non-commensurable. It follows from the last equality that every pair of elements of the chain $\left(\widehat{H}_{i}\right)_{i \in Q}$ has at least one $C B_{\varphi}^{*}$-rank distinguishing them. So the types $\left(p_{r}\right)_{r \in R}$ are pairwise inconsistent. We may complete them and build $2^{\aleph_{0}}$ complete 1-types.

Note that if $G$ is a stable group with no dense forking chain, and $f$ a definable group morphism from $G$ to $G$ with finite kernel, then $G$ and $f(G)$ have same dimension and $U_{\alpha}$-rank. This follows from the second Lascar inequality applied to $G$ and $\operatorname{Ker} G$. More generally:

LEMMA 3.11. Let $X$ and $Y$ be type-definable sets, and let $f$ be a definable map from $X$ onto $Y$ the fibres of which have no more than $n$ elements for some natural number $n$. Then $X$ and $Y$ have the same dimension, and same $U_{\alpha}$-rank for every ordinal $\alpha$.

Proof. One just needs to notice that if $q$ is some type in $X$, and if $p$ is an extension of $q$, then $p$ is a forking extension of $q$ if and only if $f(p)$ is a forking extension of $f(q)$.

Proposition 3.12. Let $G$ be a stable group without dense forking chain, and let $f$ be a definable group morphism from $G$ to $G$. If $f$ has finite kernel, its image has finite index in $G$.

Proof. Let us write $H$ for $f(G)$, and let us apply the first Lascar equality. We get $U_{\alpha}(H)+U_{\alpha}(G / H) \leq U_{\alpha}(G)$. But $H$ and $G$ have the same $U_{\alpha}$-rank after Lemma 3.11. It follows that $U_{\alpha}(G / H)$ is zero. This holds for every ordinal $\alpha$, so $\operatorname{dim}(G / H)$ is -1 . This means that $G / H$ is finite.

Remark 3.13. In Proposition 3.12, one cannot bound the index of the image of $f$ with the cardinal of its kernel. Consider for instance the superstable group $(Z,+)$, and the maps $f_{n}$ mapping $x$ to the $n$ times $\operatorname{sum} x+\cdots+x$, when $n$ ranges among natural numbers.

COROLLARY 3.14. If $K$ is an infinite weakly small field, and $L / K$ a field extension of finite degree, then $L$ has no Artin-Schreier extensions.

PRoof. If $K$ has positive characteristic $p$, let $f$ be the Artin-Schreier map from $L$ to $L$. We consider the additive structure of $L$, together with $f:$ it is an Abelian structure with weakly small universe $K$. It has no dense forking chain by Theorem 3.9. The map $f$ has finite fibres so $f(L)$ has finite additive index in $L$ by Proposition 3.12. But $K$ has no proper definable additive subgroup of finite index by Theorem 2.2, so neither has any finite Cartesian power of $K$, thus $f$ is onto. -1 
COROLLARY 3.15. The degree of an algebraic extension of an infinite weakly small field of positive characteristic $p$ is not divisible by $p$.

Proof. Let $K$ be this infinite weakly small field; it is perfect by Theorem 2.2. If there is an algebraic extension the degree of which is divisible by $p$, there is also a normal separable extension $L$ of finite degree divisible by $p$. Its Galois group has a subgroup of order $p$, the invariant field of which we note $K_{1}$. The extension $L / K_{1}$ is an Artin-Schreier extension, a contradiction.

COROLLARY 3.16. A weakly small field of characteristic two is either finite or algebraically closed.

PROOF. If it is infinite and not algebraically closed, it has a normal separable algebraic extension of finite degree. According to Corollaries 3.15 and 3.1, its Galois group neither has even order, nor is soluble, a contradiction to Feit-Thomson's Theorem.

§4. Weakly small division rings. Recall that a superstable division ring is a field [1, Cherlin, Shelah]. It is shown in [12] that a small division ring of positive characteristic is a field. It is still unknown whether this extends to weakly small division rings. In this section we show, at least, that every finitely generated algebraic closure in a weakly small division ring has finite dimension over its centre. With the previous section, this implies that a weakly small division ring of characteristic 2 is a field.

If $D$ is an infinite weakly small division ring, and $K$ a definable sub-division ring of $D$, one may view $D$ as a left or right vector space over $K$. However, we will not distinguish between the left and right $K$-dimension of $D$ thanks to:

LEMMA 4.1. If $K$ is a definable sub-division ring of $D$, and if $D$ has finite left or right dimension over $K$, then $D$ has finite right and left dimension over $K$, and those dimensions are the same. Moreover, there is a set which is both a left and right $K$-basis of $D$.

Proof. Let $f_{1}, \ldots, f_{n}$ be a left and right $K$-independent family from $D$, with $n$ maximal. Let $F_{r}$ and $F_{l}$ be the set of respectively right and left linear $K$ combinations of the $f_{i}$. If $F_{r}<D$ and $F_{l}<D$, then $F_{r} \cup F_{l}$ is a union of two proper subgroups of $D^{+}$hence $F_{r} \cup F_{l}<D$. Taking a non-zero element $f_{n+1}$ in $D \backslash F_{r} \cup F_{l}$ yields a familly $f_{1}, \ldots, f_{n+1}$ wich is both right and left $K$-independent, a contradiction with the maximality of $n$. So we may suppose that $D$ equals $F_{r}$. The group homomorphism from $D^{+}$mapping a right decomposition $\sum_{i=1}^{n} f_{i} k_{i}$ to $\sum_{i=1}^{n} k_{i} f_{i}$ is a definable embedding, hence surjective after Lemma 1.3. Thus $F_{l}, F_{r}$ and $D$ are equal.

For the next Proposition, we recall the following result:

FACT 4.2 (Herstein [4, Lemma 3.1.1]). In a division ring of positive characteristic, let $a$ be a non-central element of finite order. There exists a natural number $n$ and an element $x$ with $x a x^{-1}=a^{n}$ but $a^{n} \neq a$.

PROPOSITION 4.3. The centre of an infinite weakly small division ring is infinite.

Proof. We may assume that $D$ has positive characteristic, as this obviously holds in zero characteristic. We may also assume that $D$ is not locally finite and has an element $b$ of infinite order. It follows from Corollary 3.1 that the field $Z(C(b))$ 
has no Galois solvable extension: in particular, for every prime number $q$, there exists some non-trivial $a$ in $Z(C(b))$ with $a^{q}=1$. We claim that all those roots are in $Z(D)$. Suppose not, and let $a$ be non central with $a^{q}=1$ for a prime number q. According to Fact 4.2, there exists a natural number $n$ and an $x$ in $D$ with $x a x^{-1}=a^{n}$ but $a^{n} \neq a$. If $x$ has finite order, the division ring generated by $x$ and $a$ is finite, a contradiction to Wedderburn's Theorem. So $x$ has infinite order. Conjugating $q-1$ times by $x$, we get $x^{q-1} a x^{-q+1}=a^{n^{q-1}}=a$. Note that $x^{q-1}$ has infinite order, so $Z\left(C\left(x^{q-1}\right)\right)$ is infinite and has no proper field extension obtained by adding a radical in view of Corollary 3.1, and thus contains $x$. It follows that $a$ and $x$ commute, a contradiction.

COROLLARY 4.4. In an infinite weakly small division ring, an element and a power of it have the same centraliser.

Proor. Let $a$ be in $D$. We obviously have $C(a) \leq C\left(a^{n}\right)$. Conversely, by Proposition 4.3, the field $Z\left(C\left(a^{n}\right)\right)$ is infinite. Corollary 3.1 implies that it contains $a$.

Remark 4.5. It follows that every element having finite order lies in the centre. Similarly, for every non constant polynomial $P$ with coefficients in the centre having a soluble Galois group, $P(a)$ and $a$ have the same centraliser in $D$. If $D$ is in addition small, this holds for every non constant polynomial with coefficients in the centre.

COROLlARY 4.6. Let $\bar{a}$ be some finite tuple in the infinite weakly small division ring $D$. The sets acl $(\bar{a}), d c l(\bar{a})$ and $\bar{a}$ have the same centraliser in $D$.

Proof. The inclusions $C(\operatorname{acl}(\bar{a})) \leq C(d c l(\bar{a})) \leq C(\bar{a})$ are easy. Conversely, suppose $x$ commutes with $\bar{a}$ and let $y$ be in $\operatorname{acl}(\bar{a})$. For every natural number $m$, the elements $y^{x^{m}}$ and $y$ are conjugated by the action of the automorphisms group fixing $\bar{a}$ pointwise. So there must be two distinct natural numbers $n$ and $m$ so that $y^{x^{n}}$ and $y^{x^{m}}$ be equal: $y$ commutes with a power of $x$, hence with $x$ by Corollary 4.4 .

LEMMA 4.7. $D$ is a weakly small division ring. Let $\gamma$ stand for the conjugation map by some $a$ in $D$. For all $\lambda$ in $D$, the kernel of $\gamma-\lambda$.id is a $C(a)$-vector space having dimension at most 1 .

Proof. Let some non zero $x$ and $y$ be in the kernel of $y-\lambda . i d$. The equalities $x^{a}=\lambda x$ and $y^{a}=\lambda y$ yield $\left(y^{-1} x\right)^{a}=y^{-1} x$.

LEMMA 4.8. In a weakly small division ring of positive characteristic, for all a, every finitely generated algebraic closure $\Gamma$ containing $a$ is a finite dimensional $C_{\Gamma}(a)$-vector space.

Proof. We may assume that $a$ is non-central. We write $f$ for the endomorphism mapping $x$ to $x^{a}-x$. Let $K$ and $H$ stand for the kernel and the image of $f$ respectively. Note that $H$ does not contain 1, for otherwise there would be some $x$ verifying $x^{a}=x+1$ and $x^{a^{p}}=x+p=x$, a contradiction to Corollary 4.4. The set $H$ is a $K$-vector space so the intersection $H \cap K$ is an ideal of $K$ not containing 1 hence trivial. Consider the map induced by $f$ from $D^{+} / K$ to $D^{+} / K$ : it is injective hence surjective, so we get $D=H \oplus K$. This yields

$$
\Gamma=H \cap \Gamma \oplus K \cap \Gamma .
$$


The intersection $I$ of the sets $\lambda H \cap \Gamma$ where $\lambda$ runs over $\Gamma$ is a finite intersection, of size $n$ say: it is a left ideal of $\Gamma$, hence zero. But $H \cap \Gamma$ is a $K \cap \Gamma$-vector space having codimension 1 , so $I$ has codimension at most $n$.

THEOREM 4.9. A weakly small division ring of positive characteristic is locally finite dimensional over its centre.

Proof. Let $\Gamma$ be a finitely generated algebraic closure, and $D_{0}, \ldots, D_{n+1}$ a maximal chain of centralisers of elements in $\Gamma$ such that the chain

$$
\Gamma>D_{1} \cap \Gamma>\cdots>D_{n} \cap \Gamma>D_{n+1} \cap \Gamma
$$

be properly descending, and $D_{n} \cap \Gamma$ be minimal non commutative. The fields extensions $D_{i} \cap \Gamma / D_{i+1} \cap \Gamma$ are finite by Lemma 4.8. As $D_{n+1} \cap \Gamma$ is a field, $\Gamma$ has finite dimension over its centre, bounded by $\left[\Gamma: D_{n+1} \cap \Gamma\right]^{2}$ according to [2, Corollary 2 p.49].

COROLlaRY 4.10. A small division ring of positive characteristic is a field.

Proof. Let $\Gamma$ be the algebraic closure of a finite tuple $\bar{a}$. By Corollary 4.6, we have

$$
Z(\Gamma)=Z(C(\Gamma)) \cap \Gamma=Z(C(\bar{a})) \cap \Gamma
$$

By [24], $Z(C(\bar{a}))$ is algebraically closed. It follows that $Z(\Gamma)$ is relatively algebraically closed in $\Gamma$, so a small division ring is locally commutative, hence commutative.

COROLlARY 4.11. A weakly small division ring of characteristic 2 is a field.

Proof. Follows from Corollary 3.16 with the same proof as Corollary 4.10.

COROLLARY 4.12. Vaught's conjecture holds for the pure theory of a positive characteristic division ring.

Proof. If the theory of an infinite pure division ring has fewer than $2^{\aleph_{0}}$ denumerable models, it is small: it is the theory of a algebraically closed field, which has countably many denumerable models as noticed in [24].

In positive characteristic, we can just say the following:

Proposition 4.13. If $D$ is a small division ring, let a be outside the centre, and write $\gamma$ for the conjugation by $a$. For every non-zero polynomial $a_{n} X^{n}+\cdots+a_{1} X+a_{0}$ with coefficients in the centre of $D$, the morphism $a_{n} \gamma^{n}+\cdots+a_{1} \gamma+a_{0} I d$ is onto.

Proof. Let $K$ be the field $C(a)$. As $Z(D)$ is algebraically closed, $P$ splits over $Z(D)$. As a product of surjective morphisms is still surjective, it suffices to show the result for some irreducible $P$. Let $\lambda$ be in the centre, let $f$ be the morphism $\gamma-\lambda$.id, and let $t$ be outside the image of $f$. The map $f$ is a $K$-linear map; its kernel must be a line or a point. According to Proposition 1.7, we get $D=\operatorname{Ker} f^{m}+\operatorname{Im} f^{m}$ for some natural number $n$. Set $H$ the image of $f^{m}$, and $L$ its kernel. Note that $L$ has finite $K$-dimension. We may replace $L$ by a definable summand of $H$, and assume that $L$ and $H$ be disjoints. Let $\Gamma$ be an infinite finitely generated algebraic closure containing $t, a$, some $b$ which does not commute with $a$, and the $K$-basis of $L$. We still have

$$
\Gamma=L \cap \Gamma \oplus H \cap \Gamma .
$$

The intersection $I$ of the sets $\lambda H \cap \Gamma$, where $\lambda$ runs over $\Gamma$ is a finite intersection by Theorem 1.5: it is an ideal of $\Gamma$ which does not contain $t$, hence zero. But $H \cap \Gamma$ 
has finite $K \cap \Gamma$-codimension, hence so has $I$. According to [2, Corollary 2 p.49], we have

$$
[\Gamma: K \cap \Gamma]=\left[\Gamma: C_{\Gamma}(a)\right]=[Z(\Gamma)(a): Z(\Gamma)]<\infty
$$

But $Z(\Gamma)$ is nothing more than $Z\left(C_{D}(\Gamma)\right) \cap \Gamma$. By Corollary 4.6, $Z\left(C_{D}(\Gamma)\right)$ is an algebraically closed field so $a$ belongs to $Z(\Gamma)$, a contradiction.

COROLLARY 4.14. In a small division ring, the central algebra generated by the conjugation map by any element is a division algebra.

Proof. Set $\gamma$ a conjugation map. If $f$ and $g$ are two polynomials in $\gamma$ such that $f . g$ is zero, then either $f$ or $g$ is zero.

\section{REFERENCES}

[1] Gregory Cherlin and Saharon Shelah, Superstable fields and groups, Annals of Mathematical Logic, vol. 18 (1980), no. 3, pp. 227-270.

[2] Paul Moritz Conn, Skew field constructions, Cambridge University Press, Cambridge, 1977.

[3] DaVID Evans and Frank WaGNER, Supersimple $\omega$-categorical groups and theories, this JourNaL, vol. $65(2000)$, no. 2 , pp. $767-776$.

[4] ISRAEL N. HERSTEIN, Noncommutative rings, The Mathematical Association of America, quatrième édition, 1996.

[5] Bernhard Herwig, James G. Loveys, Anand Pillay, Predag Tanović, and Frank O. Wagner, Stable theories without dense forking chains, Archive for Mathematical Logic, vol. 31 (1992), no. 5, pp. 297-303.

[6] Ehud Hrushovski and Anand Pillay, Weakly normal groups, Logic colloquium '85 (The Paris Logic Group, editor), North-Holland, Amsterdam, 1987, pp. 233-244.

[7] Itay Kaplan, Thomas Scanlon, and Frank O. Wagner, Artin-Schreier extensions in NIP and simple fields, Israel Journal of Mathematics, vol. 185 (2011), pp. 141-153.

[8] Byunghan Kim, A note on Lascar strong types in simple theories, this Journal, vol. 63 (1998), no. 3, pp. 926-936.

[9] KRZYSZTOF KRUPIŃSKI and LUDOMIR NEWELSKI, On bounded type-definable equivalence relations, Notre Dame Journal of Formal Logic, vol. 43 (2002), no. 4, pp. 231-242.

[10] Serge Lang, Algebra, Springer-Verlag, New York, 2002.

[11] Angus Macintyre, On $\omega_{1}$-categorical theories of fields, Fundamenta Mathematicae, vol. 71 (1971), no. 1, pp. 1-25.

[12] Cédric Milliet, Small skew fields, Mathematical Logic Quarterly, vol. 53 (2007), no. 1, pp. 86 90 .

[13] - On properties of (weakly) small groups, this JouRnaL, vol. 77 (2012), pp. 94-110.

[14] Anand Piliay, Countable models of 1-based theories, Archive for Mathematical Logic, vol. 31 (1992), no. 3, pp. 163-169.

[15] Anand Pillay and Bruno Poizat, Pas d'imaginaires dans l'infini!, this Journal, vol. 52 (1987), pp. 400-403.

[16] Anand Pillay, Thomas Scanlon, and Frank O. Wagner, Supersimple fields and division rings, Mathematical Research Letters, vol. 5 (1998), pp. 473-483.

[17] BRuno PoIzat, Cours de théorie des modèles, Nur al-Mantiq wal-Ma'rifah, Villeurbanne, 1985.

[18] — Groupes stables, Nur al-Mantiq wal-Ma'rifah, Villeurbanne, 1987.

[19] - Quelques tentatives de définir une notion générale de groupes et de corps de dimension un et de déterminer leurs propriétés algébriques, Confluentes Mathematici, vol. 1 (2009), no. 1, pp. 111-122.

[20] Vera A. PUNINSKAYA, Vaught's conjecture, Journal of Mathematical Sciences (New York), vol. 109 (2002), pp. $1649-1668$. 1037 .

[21] Frank O. WAGNER, Small stable groups and generics, this JournaL, vol. 56 (1991), pp. 1026-

[22] - Quasi-endomorphisms in small stable groups, this JoURNAL, vol. 58 (1993), pp. 1044 1051.

[23] — , Stable groups, Cambridge University Press, Cambridge, 1997. 
[24] - Small fields, this JouRnaL, vol. 63 (1998), no. 3, pp. 995-1002.

[25] — Minimal fields, this JourNal, vol. 65 (2000), no. 4, pp. 1833-1835.

[26] VOLKER WEISPFENNING, Quantifier elimination for abelian structures, preprint, 1983.

UNIVERSITÉ DE LYON, UNIVERSITÉ LYON 1

INSTITUT CAMILLE JORDAN, UMR 5208 CNRS

43 BOULEVARD DU 11 NOVEMBRE 1918 69622 VILLEURBANNE CEDEX, FRANCE

(Current address) UNIVERSITÉ GALATASARAY FACULTÉ DE SCIENCES ET DE LETTRES DÉPARTEMENT DE MATHÉMATIQUES CIRAĞAN CADDESI N 36 34357 ORTAKÖY, ISTAMBOUL, TURQUIE

E-mail: milliet@math.univ-lyon1.fr 